$\xi_{p}$

\title{
The Influence of Rewards on Perceptions, Competence, and Sensory and Imaginative Immersion of Malaysian Digital Traditional Games
}

\author{
Cik Fazilah Hibadullah, Noraziah ChePa, Nur Azzah Abu Bakar and Azham Hussain \\ Human Centered Computing Research Lab, School of Computing, Universiti Utara Malaysia, 06010 Malaysia \\ *Corresponding Author Email: azham.h@uum.edu.my
}

\begin{abstract}
Rewards in games are among the essential features to attract players to engage with the game. Prominent scholars claimed that rewards have positive influences to game engagement. Despite the importance of rewards in digital games, none of the existing Malaysian digital traditional games incorporated rewards in the games. Therefore, this study is conducted to investigate the influence of rewards on digital games engagement which focusing on perceptions, competence, and sensory and imaginative immersion on one of the Malaysian digital traditional games, Congkak. The existing Congkak has been enhanced by incorporating rewards. Fifty respondents among the millennials were involved in the experiment to measure the influence of rewards using Game Experience Questionnaire (GEQ). Findings revealed that all constructs tested demonstrate positive influence of rewards on game engagement. The findings serve as a guideline to game developers to improve the games in order to foster better engagement of Malaysian digital traditional games.
\end{abstract}

Keywords: rewards, games engagement, digital games, game perceptions, Congkak.

\section{Introduction}

digital games are defined as games played on digital devices and it is an interactive applications that can be played individually or in groups. As a complement to the concept of digital games, the introduction of reward systems can be seen as a motivation and appreciation to the player [1]. Moreover, according to [2], digital games have found to be effective to increase knowledge, to support engagement, to improve problem-solving skills and to generate information processing. Digital technologies are used to enhance existing traditional games to increase pleasure when playing the games. Digital technologies have the potential to add special effects to excite players emotionally. In addition, the technologies can also help players to learn complex rules in the games [3].

Due to the advancement of technology, it is now becomes a trend among young generation to play digital games rather than traditional games. Many of the existing traditional games have started to be forgotten. Young generations are more influenced to engage with new technology using their digital gadgets such as smartphones and tablets [4]. The innovation and creativity of technology give an advantage to the players to play and compete with other players remotely. A contemporary digital game such as Minecraft is more capable to offer advantages to young generation to play in virtual groups although they are not in the same place.

Traditional games of a nation are unique and usually reflected a strong sense of the community [5]. According to [6], cultural heritage and natural history of a nation have a very high value and unique. Example of traditional games are sepak raga, guli, kasut tempurung, batu seremban and Congkak. The essential of traditional games not only play for fun and leisure activities, but it is also beneficial for learning process [4]. Conrado and his team [7] agreed that by adapting traditional approach in computer games, it can preserve the country's heritage and culture while making it interesting for the next generation. Utilization of information and communication technologies to preserve cultural heritage and natural history has been implemented by some developed countries. Other Asian countries also have a rich and diverse culture and mythologies which could be a good source of content for modern computer games [7]. Some studies revealed that digital games can be considered as a convergence of art and technology [7]. Many approaches to enhance traditional games have been used, for example Augmented Reality (AR) [3] and Neural Network (NN) [8]. Despite their importance to preserve cultural heritage, digital traditional games are far less popular than digital contemporary games [2]. There is a significant difference between the download rate of digital traditional games if compared to the download rate of contemporary versions. Taking example of Minecraft and Congkak to represent both genres, the download rate shows a significant difference. Minecraft has been downloaded for 2097676 times, while Congkak has only been downloaded for 3371 times as recorded on 28th November 2017 on Google Play Store. It shows how contemporary version is way popular than the traditional one.

One of the impeding factors is due to the lack of rewards elements. 
Very few studies have been conducted to address the issues associated to rewards in digital traditional games. Most of the contemporary games are full of excitement, interactive, challenging and therefore can attract players to the games. This article discusses the influence of rewards in Malaysian digital traditional games towards games engagement, particularly in the context of perceptions, competence, and sensory and imaginative immersion. This article is organized as follows; Section I discusses introduction of the study that describes the background and objectives. Previous studies discussing about rewards in digital games are covered in Section II. Discussions on research methodology and findings are presented in Section III and IV respectively. Finally, conclusions of the study are discussed in Section V.

\section{Rewards in Digital Games}

In the context of digital traditional games, it is crucial to provide features such as challenges and rewards to players in order to attract and make them hooked to the games. According to [9] and [10], game reward system inspires the player to have positive motivation to engage with digital games. Modern digital games provide social meaning for players to keep engaged with the digital games. Rewards system can influence players to have fun and enjoy the game [1]. David [9] stated that rewards in digital games can be scheduled in four different types based on different system of rewards; (1) fixed ratio schedule - the player receives a reward after a fixed number of action, (2) variable ratio schedule - the player receives a reward after a random number of actions, (3) fixed interval schedule - the player receives a reward after fixed interval of time, and (4) variable interval schedule - the player receives a reward after a variable interval of time. Usually, in simple digital games, one type of reward can be applied. However, in complex digital games, more reward schedules can be applied in order to provide the right level of engagement for the players. Rewards in digital games are needed to improve motivation and increase momentum and can influence players to engage with the digital game.

An interesting study on implementing rewards in traditional games has been conducted by [7]. The researchers presented two computer games that have been developed and how these can be used to exhibit the culture and art of the country and used computer games as a medium for supporting Asian culture and art. Their study investigated how engagement in digital traditional games can be influenced by rewards. The first game is based on Thailand's popular transportation, Tuk-Tuk (auto rickshaw). The game used 3D taxi simulation where the player has to pick up passengers and send them to their requested destination and receives a money as rewards. The more passengers the players send, the more rewards they will collect. While the second game is a traditional board game played with stones in Vietnam, namely $O$ An Quan (game of squares). This game is similar to Malaysia's Congkak and the Philippines' Sungka. The board design game consists of two big half circle shape called Mandarin's boxes on the left and right and 10 small squares. Five pebbles are placed in each of the 10 small squares. The more pebbles the players collect, the more rewards will be collected. Player who collects more pebbles will be announced as a winner of the game. The idea of incorporating rewards in the mentioned digital traditional games can be implemented to Malaysian Congkak.

\section{Methodology}

There are five main phases involved in conducting the study as illustrated in Figure 1.

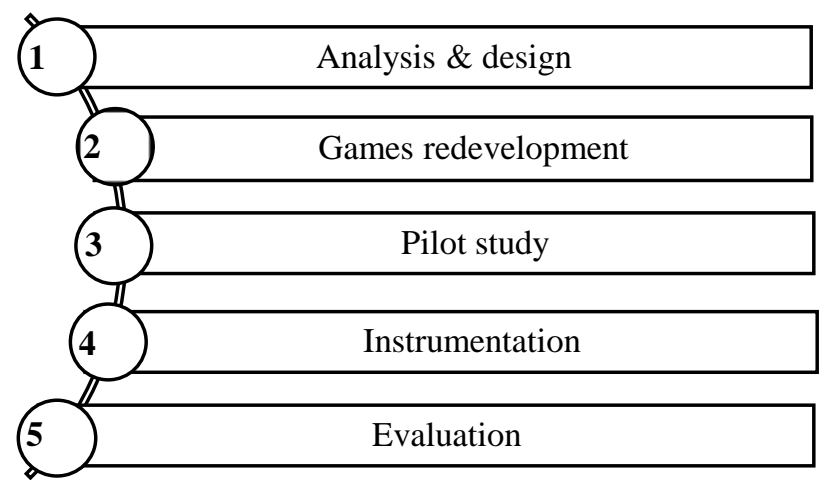

Figure 1: Research Methodology

Analysis and Design: This phase involved an extensive literature review to understand the theories of games engagement such as rewards. As a result, this phase concluded that rewards can be implemented in digital traditional games. There are some characteristics in rewards feature such as score system, item grating in rewards system and unlocking mechanisms can that attract and engage players.

Several digital games which are available from Google Play Store were studied with the aim to select the most appropriate game to be used in evaluating the influence of rewards on digital traditional game engagement. Finally, Congkak that was developed by $\mathrm{Mr}$ Shamsul Bahrin Abdul Mutalib [11] was selected to be improved due to its high download rate and good reviews and feedback.

Games redevelopment: This phase involved the redevelopment of Congkak by considering three enhancements; changing of gameplay from anti-clockwise to clockwise, adding rewards (see Figure 2) and adding flexibility to the board size from the original 7 holes to either 6,7 or 8 holes (see Figure 3).

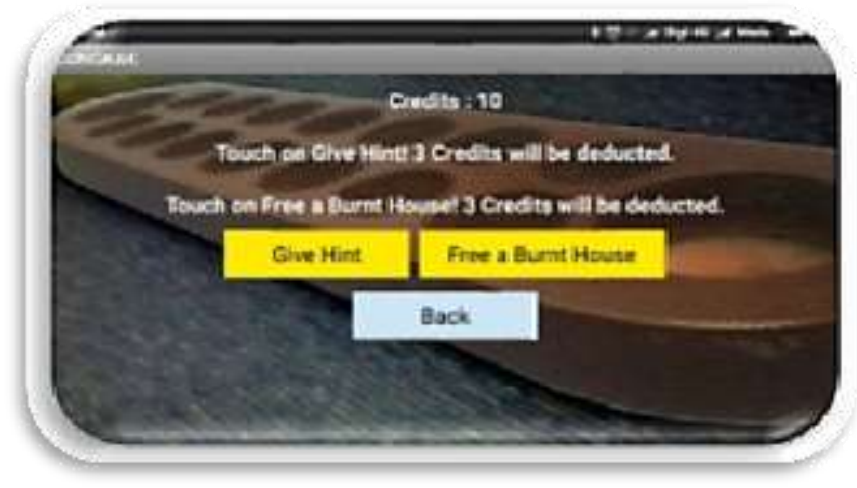

Figure 2: Rewards Credit in Congkak

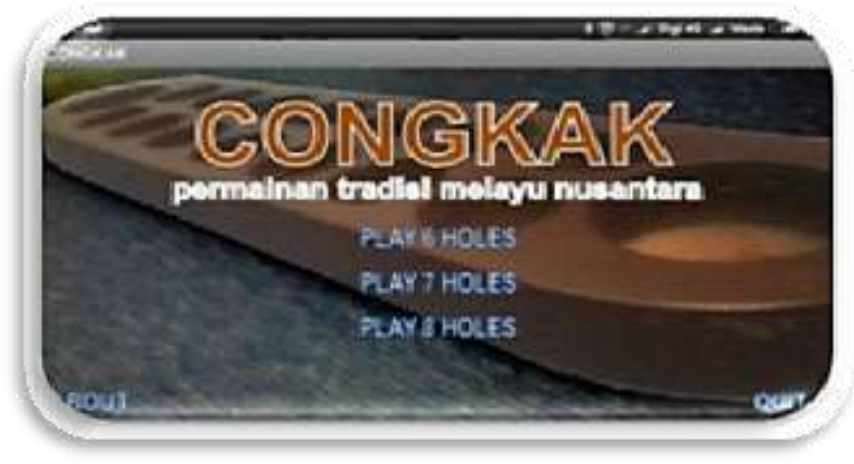

Figure 3: Choices of Board Size 
Pilot Study: Pilot study was conducted in three steps which are game demo, game experience, and systematic interview. The study involved 40 respondents among the millennials (14-34 years old). Pilot study focused on investigating perceptions towards rewards in digital traditional games in six aspects which are the importance of rewards, the necessity of rewards in each level, the necessity of rewards at difficult level, motivation, survival, and lastly the influence of rewards on game engagement. Findings from the pilot study revealed a positive perception towards rewards with regard to when rewards are needed, the importance of rewards, and role of rewards in motivating the players, survival as well as engagement to the game.

The instrument used in pilot study is further enhanced by adding two additional constructs which are competence and sensory and imaginative immersion.

Instrumentation: The instrument used in this study is divided into five sections; demographic profile of the respondent, perception towards reward in digital traditional games, competence, sensory and imaginative immersion, and open-ended questions to gather suggestions from the respondents on their preference of rewards. Section 3 and 4 of the questionnaire are adapted from Games Experience Questionnaire (GEQ)[12].

The questionnaire is designed using Likert scale ranges from 0 to 4 where 0 indicates Strongly Disagree, 1 indicates Disagree, 2 indicates Fair, 3 indicates Agree and 4 indicates Strongly Agree.

To measure perception, there are six items used (labelled as P1 to P6) as listed in Table 1.

Table 1: Items to Measure Perception towards Rewards

\begin{tabular}{|c|l|}
\hline Items & Description \\
\hline P1 & Rewards are important in playing games \\
\hline P2 & Rewards are needed at each level of the games \\
\hline P3 & Rewards only needed at difficult level \\
\hline P4 & Rewards motivate me to play \\
\hline P5 & Rewards help me to survive the game \\
\hline P6 & Rewards help me to keep playing \\
\hline
\end{tabular}

Table 2 shows three items (labelled as C1, C2, and C3) used to measure the influence of rewards on competence while Table 3 shows two items (labelled as S1 and S2) used to measure the influence of rewards on sensory and imaginative immersion.

Table 2: Items to Measure the Influence of Rewards on Competence

\begin{tabular}{|c|l|}
\hline Items & Description \\
\hline $\mathrm{C} 1$ & Rewards made me felt skilful \\
\hline $\mathrm{C} 2$ & Rewards made me felt successful \\
\hline $\mathrm{C} 3$ & Rewards made me fast at reaching the game's targets \\
\hline
\end{tabular}

Table 3: Items to Measure the Influence of Rewards on Sensory and Imaginative Immersion

\begin{tabular}{|c|l|}
\hline Items & Description \\
\hline S1 & Rewards made me interested in the game's story \\
\hline S2 & Rewards made me felt imaginative \\
\hline
\end{tabular}

Evaluation: The evaluation of the enhanced digital Congkak involved 50 respondents among the millennials. Similar to processes involved in pilot study, the assessment involved three steps; game demo, game experience, and systematic interview. Prior to game demo, the enhanced digital Congkak was installed on tablet. Researchers began by briefly explained all the functions and demonstrated how rewards work in Congkak. During game experience, respondents were given opportunities to play the game and gain experience with the game. The final step is systematic interview which involved interview and filling in the questionnaire.

\section{Findings}

This section discusses analysis and findings from the data gathered during evaluation phase. The discussion of the findings is divided into five sections; analysis of demographics of the respondents, analysis of perception on rewards, analysis of how rewards influence competence, analysis of how rewards influence sensory and imaginative immersion, and analysis of the suggestion from the respondents. Data were analyzed using frequency analysis and mean score. Descriptive analysis has been used to describe the data. Analysis of Demographic of the Respondents: Table 4 lists the demographic profile of respondents. This table is divided by age, gender, and frequency of playing the game.

Table 4: Demographic Profile of Respondents

\begin{tabular}{|l|c|c|}
\hline & Frequency & $\begin{array}{c}\text { Percentage } \\
(\%)\end{array}$ \\
\hline Age & 20 & 40 \\
\hline $20-25$ & 25 & 50 \\
\hline $26-29$ & 5 & 10 \\
\hline Above 30 & 21 & 42 \\
\hline Gender & 29 & 58 \\
\hline Male & 21 & 42 \\
\hline Female & 22 & 44 \\
\hline Frequency of playing game & 7 & 14 \\
\hline Below 1 hour & \multicolumn{1}{|l}{} \\
\hline 1-4 hour &
\end{tabular}

$50 \%$ of the respondents are between the age of 26 to 29 years old, while $40 \%$ are between 20 to 25 years old. The remaining $10 \%$ are those aged more than 30 years old. In terms of gender, out of 50 respondents, $58 \%$ of them are female and $42 \%$ are male. In terms of the frequency of playing the game, $42 \%$ respondents play games is less than one hour per day, $44 \%$ play between one to four hour per day and $14 \%$ play more than 4 hours per day. This information indicates that all respondents have experienced playing digital games.

Analysis of Perception of Rewards: Figure 4 represents mean score for each of the six items used in measuring perception towards rewards. Overall score shows that all items have positive perceptions on rewards towards game engagement.

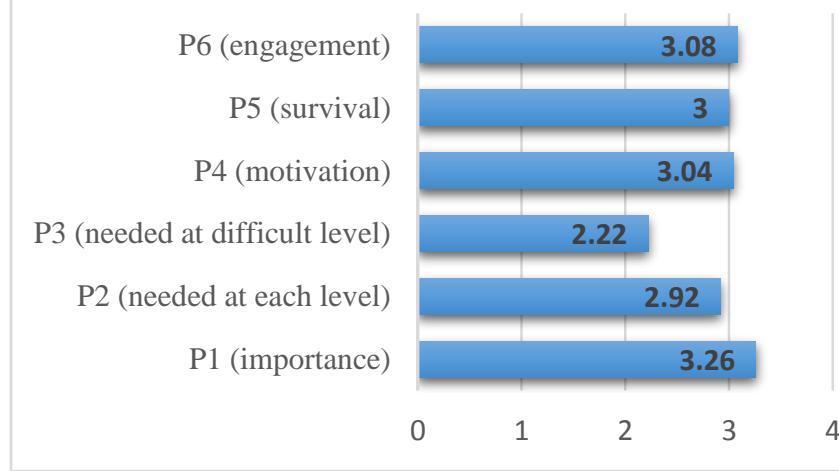

Figure 4: User Perception of Rewards in Digital Traditional Games

The highest mean score belongs to P1 (rewards are important in playing games) with 3.26 , followed by P6 (rewards help players to keep playing) with 3.08 and P4 with 3.04 while P3 (rewards only needed at difficult level) has the lowest score of average 2.22. This result demonstrates that rewards are important element in playing digital games, and to keep them playing. Finding from this study shows that rewards are not only needed at the difficult level but 
should be applied at all levels. The findings are consistent with the studies from $[4,6,10]$.

Analysis of How Rewards Influence Competence: Competence level indicates sufficiency of skills that enable the player to play the games. Mean score for each of the items used to measure the influence of rewards on competence is shown in Figure 5.

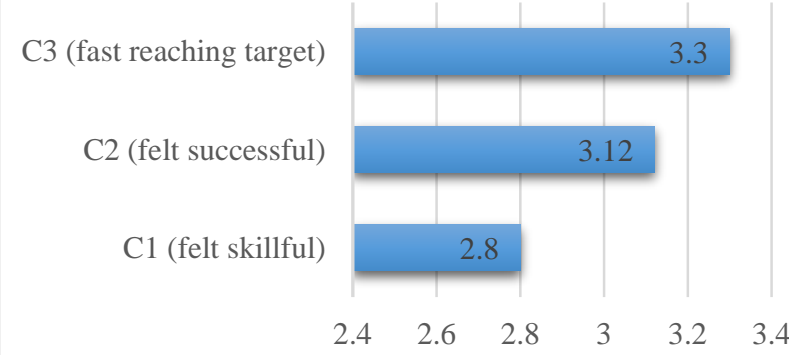

Figure 5: The Influence of Rewards on Competence

For each of the items being measured, mean score is above 3 except the mean score for items $\mathrm{C} 1$ (felt skilful) which is 2.8. It indicates that majority of the respondents have positive response towards the influence of rewards on competence level.

Mean score for items C3 (fast reaching target) and C2 (felt successful) are 3.3 and 3.12 respectively. Majority of the respondents agreed that rewards made them fast at reaching the game's targets and rewards made them felt successful. In addition, rewards are also perceived as factors to make them felt skilful (C1) with the mean score of 2.8. Findings of the study are consistent with [10] which claimed that being good at games is one way to increase player's competence level.

Analysis of How Rewards Influence Sensory and Imaginative Immersion: Sensory and Imaginative Immersion items measure the acceptance and feeling of respondents while playing the games [12]. Mean scores for these items are shown in Figure 6.

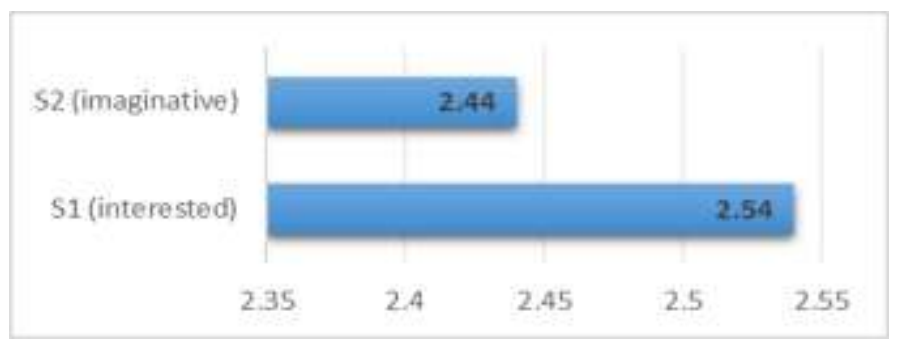

Figure 6: The Influence of Rewards on Sensory and Imaginative Immersion

Mean score for items S1 (interested in the game's story) and S2 (felt imaginative) are 2.54 and 2.44 respectively. Respondents agreed that while playing the enhanced Congkak, they felt that rewards made them more interested in the game's story (S1) and felt imaginative (S2). This could be the reasons for respondents to keep playing the game.

Analysis of Rewards Suggestions: The last section of the questionnaire used an open-ended type of question to ask the respondents to provide a suggestion on what kind of reward they favour in the digital game to improve engagement of the game. Although not all of the respondents provide their suggestion in the questionnaire, those respondents who gave recommendation able to provide worthy suggestions as shown in Table 5.
Table 5: Suggestion of Rewards

\begin{tabular}{|c|l|}
\hline Suggestion & Description \\
\hline S1 & Money \\
\hline S2 & Token to upgrade to the difficult level \\
\hline S3 & Extra life to move to the next level \\
\hline S4 & Point \\
\hline S5 & Real voucher \\
\hline S6 & Provide better facilities of the game \\
\hline S7 & Recognition \\
\hline S8 & Real reward \\
\hline S9 & Coin reward \\
\hline S10 & Fame \\
\hline S11 & House \\
\hline S12 & Virtual gold \\
\hline
\end{tabular}

Data shows that S1 (money) turned out to be the highest wanted reward followed by S2 (token to upgrade to the difficulty level) compared to other forms of rewards.

\section{Conclusion}

Rewards are the factor that can attract and engage players towards digital games. It will inspire and motivate player at certain difficulty level to keep engaged with the games. In this study, the enhanced Congkak with rewards has been developed and used as a tool to evaluate the influence of rewards in digital traditional games.

Findings showed that all constructs that have been tested show positive influences on game engagement. This study is anticipated to be beneficial and useful to many parties. The findings can be used as guidelines for new psychologists to acquire more understanding pertaining to games engagement through some experiments on digital environments. This study can also be beneficial to game developers to develop more entertaining digital games with rewards to attract players to keep them engaged with the games. Hence, it is suggested that future development of digital traditional games should include few types of rewards as suggested by respondents in this study.

\section{Acknowledgment}

This research is funded by Universiti Utara Malaysia (UUM) through University grant [SO code: 13725 (2017)]. Authors fully acknowledged UUM for the approved fund which makes this important research viable and effective. Credit also goes to the game developer, Mr Shamsul Bahrin Abd Mutalib and research assistant, Ms Nur Intan Syafiqah Abdullah.

\section{References}

[1] H. Wang and C.-T. Sun, "Game Reward Systems: Gaming Experiences and Social Meanings," in Proceedings of DiGRA '11, The Netherlands, 2011, pp. 1-12.

[2] N. ChePa, N. A. Abu Bakar, and A. Mohd, "Usability Evaluation of Digital Malaysian Traditional Games," Jurnal Teknologi, vol. 77, pp. $85-90,2015$.

[3] H. Sakuma, T. Yamabe, and T. Nakajima, "Enhancing Traditional Games with Augmented Reality Technologies," presented at the 9th International Conference on Ubiquitous Intelligence and Computing and 9th International Conference on Autonomic and Trusted Computing, Fukuoka, Japan, 2012

[4] N. ChePa and W. A. J. Wan Yahaya, "Reality and Challenges of Malaysian Digital Traditional Games," Journal of Engineering Science and Technology, pp. 209 - 218 April 2017.

[5] N. ChePa, A. Alwi, A. Mohamed Din, and S. Mohammad, "Digitizing Malaysian Traditional Game: e-Congkak," presented at the 
Knowledge Management International Conference (KMICe), Malaysia, 2014.

[6] Zainal A. Hasibuan, Yugo K.Isal, Baginda Anggun N.C., Mubarik Ahmad, and Nungki Selviandro, "Preservation of Cultural Heritage and Natural History through Game Based Learning," International Journal of Machine Learning and Computing, vol. 1, pp. 460-465, 2011.

[7] J. Conrado R. Ruiz, S. Chodnok, N. H. Salimi, and P. T. Ha, "Computer Games: A New Medium for Traditional Asian Art," International Journal of Information Processing and Management, vol. 1, pp. 91-99, 2010.

[8] N. ChePa, A. Alwi, A. M. Din, and S. Mohammad, "The Application of Neural Networks and Min-Max Algorithm in Digital Congkak," in 4th International Conference on Computing and Informatics, Sarawak, Malaysia, 2013, pp. 222-227.

[9] L. David. (2016), Game Reward Systems. Learning Theories. Available: https://www.learning-theories.com/game-rewardsystems.html

[10] I. Iacovides, "Digital Games: Motivation, Engagement and Informal Learning," PhD, Institute of Educational Technology The Open University United Kingdom, 2012.

[11] S. B. Abd Mutalib, "Congkak Game," ed, 2015.

[12] W. A. IJsselsteijn, Y. A. W. de Kort, and K. Poels, "The Game Experience Questionnaire," ed. Eindhoven: Technische Universiteit Eindhoven, 2013. 